Research Article

\title{
Research on Torque Distribution of Four-Wheel Independent Drive Off-Road Vehicle Based on PRLS Road Slope Estimation
}

\author{
Hongwei Ling $\mathbb{D}^{1}$ and Bin Huang $\mathbb{D}^{1,2}$ \\ ${ }^{1}$ Hubei Key Laboratory of Advanced Technology for Automotive Components, Wuhan University of Technology, \\ Wuhan 430070, China \\ ${ }^{2}$ Hubei Collaborative Innovation Center for Automotive Components Technology, Wuhan University of Technology, \\ Wuhan 430070, China \\ Correspondence should be addressed to Bin Huang; huangbinbi@163.com
}

Received 20 May 2021; Accepted 17 August 2021; Published 13 September 2021

Academic Editor: Xianjian Jin

Copyright (c) 2021 Hongwei Ling and Bin Huang. This is an open access article distributed under the Creative Commons Attribution License, which permits unrestricted use, distribution, and reproduction in any medium, provided the original work is properly cited.

\begin{abstract}
In view of the high difficulty in coupling of various electric vehicle parameters, intractable parameter estimation, and unreasonable distribution of vehicle driving torque, the four-wheel hub motor is applied to drive electric vehicles, which can instantly obtain the torque and speed of the hub motor and achieve precise control of the torque of each wheel. According to the vehicle longitudinal dynamics model, a progressive RLS (PRLS) algorithm for real-time estimation of vehicle mass and road gradient is proposed. Meanwhile, by means of taking the longitudinal acceleration of the vehicle and the road gradient obtained from the estimation algorithm as the parameter of the torque distribution at the front and rear axles, a dynamic compensation and distribution control strategy of the front and rear axle torques is designed. Moreover, based on hardware-in-the-loop real-time simulation and realvehicle tests, the effectiveness of the proposed estimation algorithm and the rationality of the real-time distribution control strategy of driving torque are verified.
\end{abstract}

\section{Introduction}

With continuous progress of automobile industry, in addition to the gradually highlighted issues such as energy, environmental protection, and safety, the electric vehicle has also become the focus of attention, in which hub motor drive is an important direction for the future electric vehicle development [1]. By means of directly installing the drive motor into wheel hub to integrate the power system, transmission system, and braking system, the hub motordriven vehicle not only greatly reduces mechanical transmission components but also simplifies the vehicle structure and lowers the entire vehicle mass. At the same time, hub motor creates a more flexible driving mode so that the fourwheel drive electric vehicle can easily switch between twowheel and four-wheel drive modes, and the front and rear axle torque distribution ratio control is more precise and flexible. Besides, compared with the internal combustion engine, hub motor can further improve the vehicle efficiency, making electric drive vehicles advantaged in active safety and energy saving control. Moreover, based on realtime vehicle dynamic information feedback, accurate identification of more parameters and precise control of more variables can be achieved [2].

At present, the algorithms for vehicle parameter estimation, mainly include least squares method, least squares method with forgetting factor, Kalman filter, extended Kalman filter, and adaptive Kalman filter. In addition, sliding mode observers, fuzzy observers, the nonlinear observer derived from Lyapunov theory, and the neural network algorithm have also been applied to a certain extent [3]. Literature [4] is put forward to estimate vehicle state and adhesion coefficient while using dual extended Kalman filter. Literature [5] realized the identification of battery model parameters by the least square method and achieved and used more accurate estimate of battery SOC through the 
genetic particle filter algorithm. The above parameter estimation or identification methods are the approaches commonly used in the domestic and foreign literature with better estimation effect. However, due to high complexity and many input variables, the above estimation algorithms generally have poor real-time calculation, choosing a parameter estimation algorithm that can meet the needs of vehicle control with high accuracy, low input variable requirements, and fast calculation speed which is quite significant.

The reasonable distribution of vehicle driving torque can comprehensively improve various performances such as vehicle power, maneuverability, steering, and safety [6-11]. According to the four-wheel drive vehicle driving torque distribution control strategy in literature [12], the driving condition is divided into straight driving and turning driving from the perspectives of improving vehicle dynamics and stability. In the straight driving condition, the vertical load transfer of vehicle front and rear axles is determined through longitudinal acceleration, and the vertical load ratio of front and rear axles is used as the driving torque distribution ratio so as to determine the amount of driving torque distributed to the rear wheels. In the turning driving condition, for the sake of ensuring good steering and stability of the whole vehicle, under the driving torque distribution control strategy with the given transmission system, the driving force is distributed from the front wheels to the rear wheels to reduce the tendency of understeer trend [13]. In highspeed operating conditions, the torque distribution control of four-wheel independent drive vehicles is prone to robust stability problems. Literature $[14,15]$ designed a gainscheduled robust controller using the linear parameter varying (LPV) system, which can not only obtain strong robustness but also reduce the order of controller to address the vehicle handling stability control considering system uncertainties. Because the power system of the hub motordriven vehicle has undergone fundamental changes, the traditional driving torque control strategy cannot be fully applied to the new hub motor vehicle. In this consideration, it is necessary to improve and optimize driving torque distribution control strategy by virtue of hub motor.

From the perspective of vehicle dynamics, vehicle mass, road adhesion coefficient, road gradient, and vehicle longitudinal acceleration are the key parameters involving the distribution of vehicle driving torque [16]. Due to the different weights of occupants and loaded goods each time when driving the vehicle, the vehicle mass is an indispensable factor in the driving torque distribution of the front and rear axles. Besides, on the premise of obtaining vehicle longitudinal acceleration, road gradient, and vehicle mass, the real-time distribution of the torque of four-wheel hub motors at the front and rear axles can be realized.

Considering that the RLS estimation algorithm requires few parameters and has good real-time performance, a progressive RLS estimation algorithm for the four-wheel hub motor vehicle is proposed in this paper. On the one hand, the algorithm can estimate the quality of the vehicle and the road slope with the least information from the on-board sensors, and on the other hand, it introduces a progressive algorithm to further improve the accuracy and stability of the estimation algorithm which realizes the accurate estimation of vehicle mass and road gradient. On this basis, a four-wheel hub motor torque dynamic distribution control strategy is proposed by utilizing the estimated parameters and the vehicle longitudinal acceleration proposed. Based on the equal distribution of front and rear axle driving torques, vehicle longitudinal acceleration and road gradient are studied comprehensively, and the front and rear axle driving torques are redistributed as well as dynamically compensated to adapt to different driving conditions and fully exert the strengths of the hub motor-driven electric vehicle.

\section{Design of Progressive RLS Parameter Estimation Algorithm}

Compared with traditional vehicles, the proposed fourwheel hub motor electric vehicle, instead of using the engine and gearbox, adopts a wheel reducer at the reduction ratio of $i_{0}=5$.

The longitudinal dynamics model in the traditional vehicle is shown in the following formulas:

$$
\begin{aligned}
F_{t} & =F_{f}+F_{i}+F_{w}+F_{j}, \\
\frac{T_{t q} i_{g} i_{0} \eta_{T}}{r} & =G f \cos \alpha+G \sin \alpha+\frac{C_{D} A}{21.15} u_{a}^{2}+\delta m \frac{\mathrm{d} u}{\mathrm{~d} t} .
\end{aligned}
$$

As can be learned from the driving mode of the hub motor-driven vehicle, the longitudinal dynamics model of the proposed vehicle is shown in the following formula:

$$
\frac{T_{t q} i_{0} \eta_{T}}{r}=G f \cos \alpha+G \sin \alpha+\frac{C_{D} A}{21.15} u_{a}^{2}+m \frac{\mathrm{d} u}{\mathrm{~d} t},
$$

where $i_{0}$ is the reduction ratio of the wheel reducer and $T_{t q}$ is the sum of the torques of four hub motors.

In the field of parameter real-time estimation and identification, the least square method has been well promoted and applied, of which RLS is a recursive form. For a single-input-single-output system (SISO), the adoption of algorithm can achieve small calculation, quick convergence speed, and fast convergence of parameter identification without needing any given condition so that the real-time and accuracy requirements in vehicle control system can be met.

The idea of the recursive identification algorithm can be summarized as follows:

New parameter estimate $=$ old parameter estimate + correction term

That is, the new recursive parameter estimates are iterated based on the old recursive estimates.

For an AR (n) (n-order autoregressive) model [17],

$$
y(t)=a_{1} y(t-1)+\cdots+a_{n} y(t-n)+\varepsilon(t),
$$

where $\varepsilon(t)$ is the zero mean, variance is white noise with $\sigma_{\varepsilon}^{2}>0, y(t)$ is the observation, and the order is known at $n$.

Its parameter estimation through the recursive least square method is expressed as follows: 


$$
\begin{aligned}
& \hat{\theta}(t+1)=\widehat{\theta}(t)+K(t+1)\left[y(t+1)-\varphi^{T}(t+1) \hat{\theta}(t)\right] \\
& K(t+1)=\frac{P(t) \varphi(t+1)}{1+\varphi^{T}(t+1) P(t) \varphi(t+1)}, \\
& P(t+1)=P(t)-\frac{P(t) \varphi(t+1) \varphi^{T}(t+1) P(t)}{1+\varphi^{T}(t+1) P(t) \varphi(t+1)}
\end{aligned}
$$

The vehicle mass is a parameter that does not change with time after the start of driving, but the road gradient is a time-varying parameter, so $t$ general RLS and exponentially weighted RLS are applied to separately identify the vehicle mass and road gradient online. When identifying multiple parameters with the recursive least square method, the identification results are affected by different parameter identification sequences. Regarding the identification sequence, an initial value close to the true value can be selected as vehicle mass when initializing the algorithm while the road gradient at the start is unknown. In this consideration, this paper proposes a progressive RLS estimation algorithm that estimates the road gradient based on the initial value of vehicle mass and then returns the estimated road gradient to the mass estimation module to estimate the vehicle mass in real-time. The logic diagram of this estimation algorithm is shown in Figure 1.

The longitudinal dynamics model of the vehicle is transformed as follows:

$$
\frac{T_{t q} i_{0} \eta_{T}}{r}-\frac{C_{D} A}{21.15} u_{a}^{2}-\left(m \frac{\mathrm{d} u}{\mathrm{~d} t}\right)=m(g f \cos \alpha+g \sin \alpha) .
$$

Let $\quad y=\left(T_{t q} i_{0} \eta_{T} / r\right)-\left(C_{D} A / 21.15\right) u_{a}^{2}-(m(\mathrm{~d} u / \mathrm{d} t))$, $\varphi=m$, and $\theta=g f \cos \alpha+g \sin \alpha$.

Then, the above formula becomes

$$
Y=\varphi \theta
$$

where $\theta$ is the expression containing the road gradient variable.

Therefore, the RLS algorithm recursive expression for gradient estimation is shown in (8)-(10). The selection range of forgetting factor is $[0,1]$. In view that the road gradient changes constantly and the old observation data have small influence on new parameters, the forgetting factor $\lambda=0.9$ is selected in this paper:

$$
\begin{aligned}
& K(k)=\frac{P(k-1) \varphi(k)}{\lambda+\varphi^{T}(k) P(k-1) \varphi(k)} \\
& \widehat{\theta}(k)=\hat{\theta}(k-1)+K(k)\left[y(k)-\varphi^{T}(k) \hat{\theta}(k-1)\right],
\end{aligned}
$$

$$
\begin{aligned}
P(k) & =P(k-1)-\frac{P(k-1) \varphi(k) \varphi^{T}(k) P(k-1)}{1+\varphi^{T}(k) P(k-1) \varphi(k)} \\
& =\frac{\left(1-K(k) \varphi^{T}(k)\right) P(k-1)}{\lambda} .
\end{aligned}
$$

In the above expression, $k$ represents the current sampling time and $k-1$ represents the previous sampling time. During the algorithm calculation, the least square gain is firstly calculated by formula (9), and then $\widehat{\theta}(k)$ and $\mathbf{P}(k)$ are calculated. The corresponding estimated value of road gradient can be obtained from the following formula:

$$
\alpha=\frac{\arccos \left\{(\theta / g)-\sqrt{1+f^{2}-\left(\theta^{2} / g^{2}\right)}\right\}}{1+f^{2}} .
$$

The gradient estimation value, after being obtained, is taken as the input variable of mass estimation module for estimating the whole vehicle mass.

By transforming the vehicle longitudinal dynamics expression,

$$
\frac{T_{t q} i_{0} \eta_{T}}{r}-\frac{C_{D} A}{21.15} u_{a}^{2}=m\left(g f \cos \alpha+g \sin \alpha+\frac{\mathrm{d} u}{\mathrm{~d} t}\right) .
$$

Let

$$
y_{1}=\left(T_{t q} i_{0} \eta_{T} / r\right)-\left(C_{D} A / 21.15\right) u_{a}^{2},
$$
$(g f \cos \alpha+g \sin \alpha+(\mathrm{d} u / \mathrm{d} t))$, and $\theta_{1}=m$;

$$
y_{1}=\varphi_{1} \theta_{1} \text {. }
$$

Then, expression of the recursive least squares method for vehicle mass estimation can be obtained as follows:

$$
\begin{aligned}
K(k) & =\frac{P(k-1) \varphi_{1}(k)}{1+\varphi_{1}^{T}(k) P(k-1) \varphi_{1}(k)} \\
\widehat{\theta}_{1}(k) & =\widehat{\theta}(k-1)+K(k)\left[y_{1}(k)-\varphi^{T}(k) \widehat{\theta}_{1}(k-1)\right], \\
P(k) & =\left(1-K(k) \varphi_{1}^{T}(k)\right) P(k-1) .
\end{aligned}
$$

Formulas (14)-(16) help obtain the real-time estimation of vehicle mass, formula (15) is the update of the least square gain, and formula (16) is the update of the error covariance.

\section{Design of Dynamic Self-Compensation Torque Distribution Control Strategy}

The force analysis of the hub motor-driven vehicle is shown in Figure 2.

According to the vehicle theoretical dynamics formula [18], through taking the moment of each force acting on the vehicle to the contact center of the front and rear wheels and 


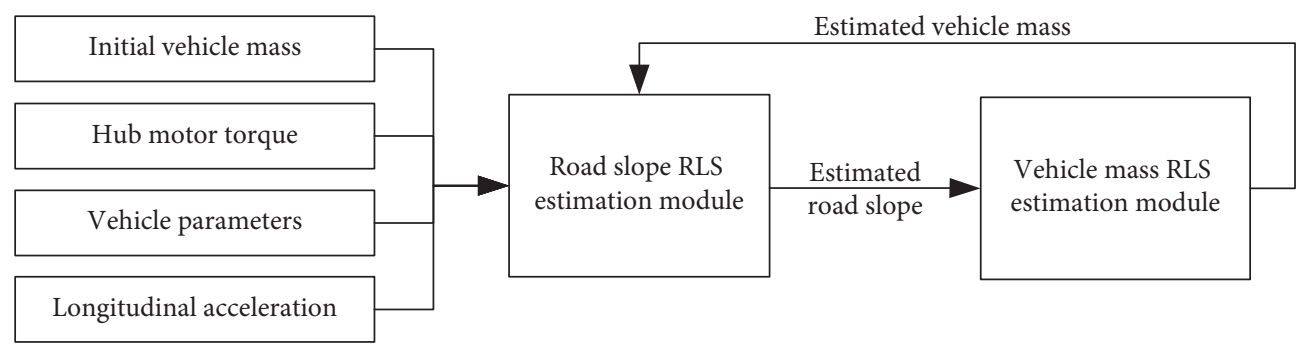

FIgURE 1: Logic diagram of the estimation algorithm.

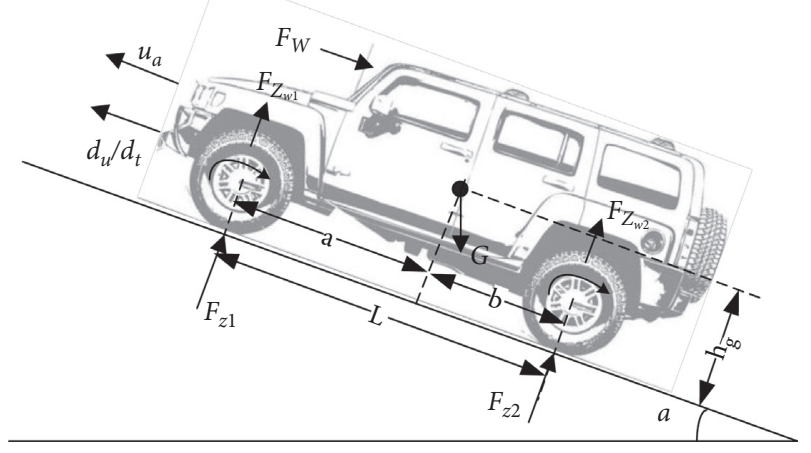

FIgURE 2: Vehicle climbing force analysis.

the road surface, the normal reaction force of the front and rear axles can be expressed as follows:

$$
\begin{aligned}
F_{Z 1}= & G\left(\frac{b}{L} \cos \alpha-\frac{h_{g}}{L} \sin \alpha\right)-\left(\frac{G}{g} \frac{h_{g}}{L}-\frac{\sum I_{w}}{L r}\right) \frac{\mathrm{d} u}{\mathrm{~d} t} \\
& -F_{Z w 1}-G \frac{r f}{L} \cos \alpha \\
F_{Z 2}= & G\left(\frac{a}{L} \cos \alpha+\frac{h_{g}}{L} \sin \alpha\right)+\left(\frac{G}{g} \frac{h_{g}}{L}-\frac{\sum I_{w}}{L r}\right) \frac{\mathrm{d} u}{\mathrm{~d} t} \\
& -F_{Z w 2}+G \frac{r f}{L} \cos \alpha .
\end{aligned}
$$

After analyzing the normal reaction force components of the proposed hub motor-driven vehicle, the rotating mass inertial resistance couple moment, rolling resistance couple moment, and air lift can be ignored under certain conditions, and the front, so that the dynamic ground normal reaction force of the front and rear axles is as follows:

$$
\begin{aligned}
& F_{Z 1}=G\left(\frac{b}{L} \cos \alpha-\frac{h_{g}}{L}\right)-\left(m \frac{h_{g}}{L}\right) \frac{\mathrm{d} u}{\mathrm{~d} t} \\
& F_{Z 2}=G\left(\frac{a}{L} \cos \alpha+\frac{h_{g}}{L} \sin \alpha\right)+\left(m \frac{h_{g}}{L}\right) \frac{\mathrm{d} u}{\mathrm{~d} t}
\end{aligned}
$$

Compared with the vehicle longitudinal acceleration, the road gradient changes slowly. Therefore, in the process of dynamic distribution of the front and rear axle driving torque, the change in the front and rear axle loads under road gradient change is used as a benchmark, and that under the vehicle longitudinal acceleration is taken as real-time dynamic compensation. According to the estimated vehicle mass and road gradient value, different front and rear axle driving torque distribution benchmarks can be obtained. At the same time, the vehicle longitudinal acceleration is obtained through the longitudinal accelerometer, and the dynamic compensation of the front and rear axle driving torque is performed accordingly $[7,19]$.

In order to prevent the jump of driving torque of the front and rear axles under the conditions of small acceleration and gradient, the gradient threshold $i_{0}$ and acceleration threshold $a_{0}$ are designed. When the gradient reaches the threshold, the new front and rear axle driving torque distribution relationship is used for the secondary distribution of driving torque; on this basis, the vehicle longitudinal acceleration signal is further collected, and dynamic compensation of the driving torque of front and rear axles based on the longitudinal acceleration is performed when the acceleration reaches the set threshold. This torque distribution control strategy not only considers the changes in driving conditions but also ensures the smooth output of torque.

The gradient threshold point is determined according to the proportional relationship of the static normal reaction force of the front and rear axles under different road gradients [20]. According to the automobile normal mathematical model established by (18) and (19), when the road gradient is $16^{\circ}$, the static normal reaction force ratio of the front and rear axles is $46: 54$. In order to make the selected torque distribution ratio adapt to more road conditions, the front and rear axle driving torque distribution ratio is determined at $40: 60$ when the road gradient is $\geq 16^{\circ}$; the front and rear axle static normal reaction ratio is determined at $38: 62$ when the road gradient is $26^{\circ}$. Based on the same selection principle, the ratio of the front and rear axle driving torque distribution is determined at 30:70 when the road gradient is $\geq 26^{\circ}$.

The driving torque output control logic of the vehicle front and rear axles is shown in Figure 3.

\section{Hardware-in-the-Loop Simulation and Actual Vehicle Test Verification}

4.1. Hardware-in-the-Loop Real-Time Simulation Test. With the purpose to verify the effectiveness of the proposed mass and gradient estimation algorithm, as well as the feasibility of the dynamic self-compensation distribution 


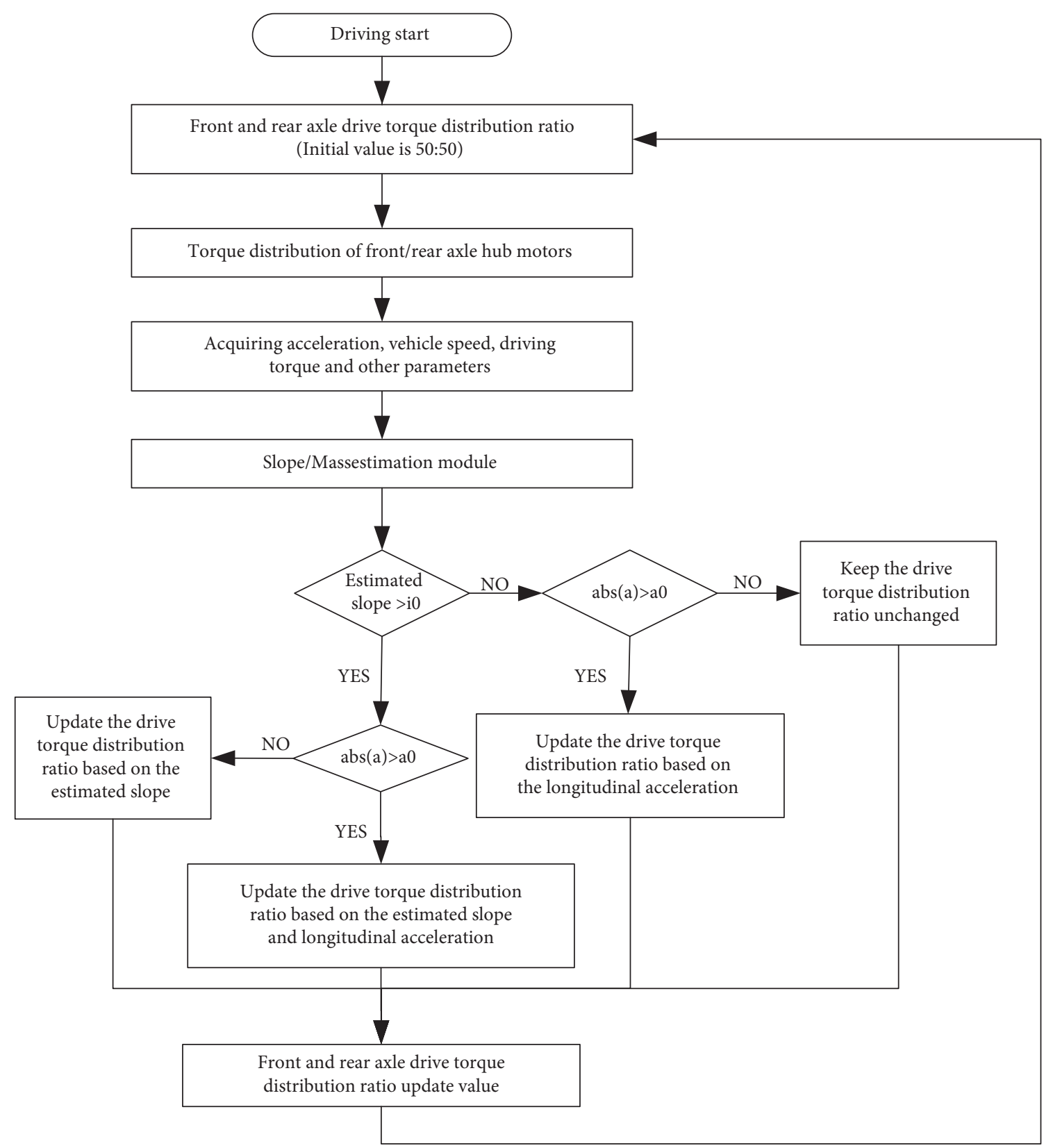

FIgURE 3: The driving torque output control logic.

control strategy of the front and rear axle driving torque, the vehicle control strategy model is established in Matlab/ Simulink. Among them, the mass and slope estimation module uses S_Function to write the estimation algorithm program, which realizes the iterative process of the progressive RLS algorithm. Then, one-key automatic code generation is used to download the control strategy model to the actual vehicle control unit (VCU). In dSPACE-ASM, a vehicle dynamics model of four-wheel hub motor-driven full-size SUV is built, and it communicates with the VCU through the CAN bus. The hardware-in-the-loop simulation platform is shown in Figure 4.

The parameter estimation simulation results are under the condition of $50 \%$ of road gradient. At the same time, the impact of the torque dynamic self-compensation distribution on the climbing performance of the vehicle is verified. The front and rear axle torques are equally distributed as a comparative experiment, and the simulation road adhesion coefficient is set to 0.8 . The vehicle starts to accelerate by stepping on the accelerator pedal at $50 \%$ on a flat road and keeps the accelerator pedal fully open after touching the slope in the $4.2 \mathrm{~s}$. The simulation results of vehicle mass and road slope estimation are shown in Figure 5.

The proposed progressive least square method can achieve real-time estimation of road gradient and vehicle mass. As can be observed from the results of joint simulation, the actual vehicle mass is $2530 \mathrm{~kg}$, and the estimated mass quickly converges near the true value; the actual value of road gradient is $25^{\circ}-28^{\circ}$; the estimated gradient quickly 


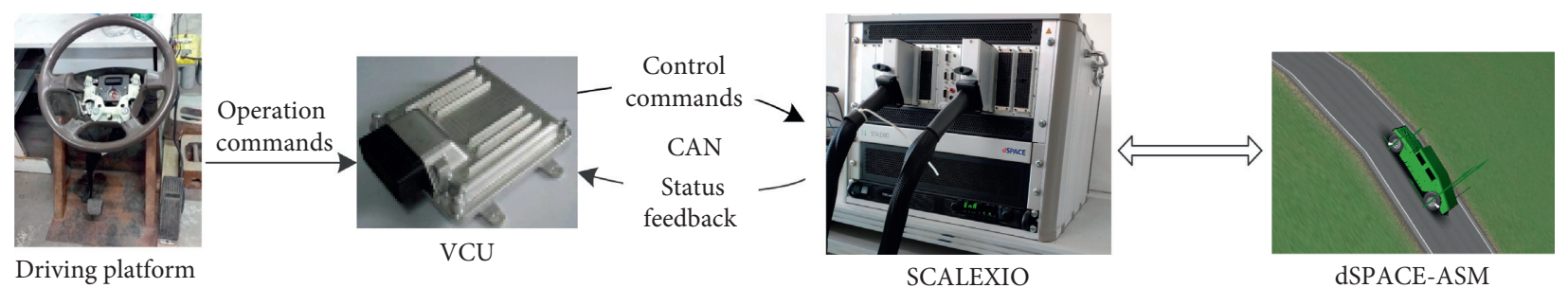

FIgURE 4: Hardware-in-the-loop real-time simulation platform.

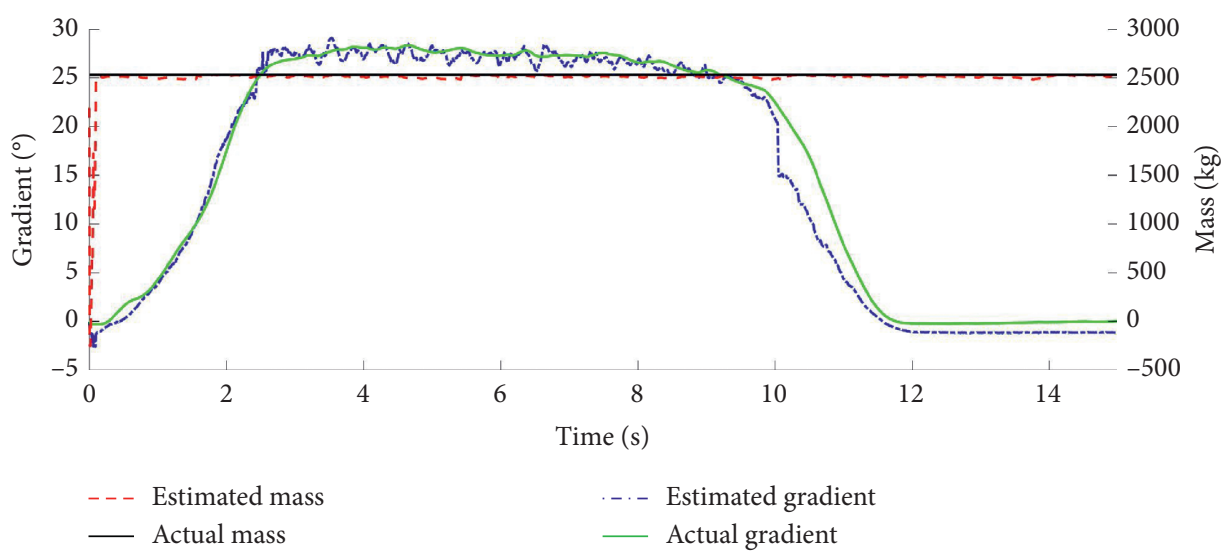

FIgURE 5: Estimation results of vehicle mass and road slope.

converges to the actual value and maintains the same trend as the actual value. The error of gradient estimation is within $2^{\circ}$, and the mass estimation error is within $10 \mathrm{~kg}$. The algorithm has high estimation accuracy, fast convergence speed, and good stability.

The torque dynamic self-compensation distribution result is shown in Figure 6.

Figures 6(a) and 6(b) show that, at $4.2 \mathrm{~s}$, the driver recognized the climb and stepped on the pedal at full throttle. The VCU sends a command to switch the motor controller to peak torque operation mode, and the motor output torque increases rapidly. Figures 6(c) and 6(d) show that when the car is running on a slope, the front and rear axle loads are shifted due to the slope of the road surface. The normal load of the front axle is greatly reduced. When the torque of each axle is evenly distributed, the maximum wheel can use the road grip which is less than the expected value of the wheel torque, the wheels slip, the motor output torque is reduced, and the vehicle traction is insufficient. Therefore, the vehicle is decelerating on the slope, and the final speed drops to zero. However, the dynamic allocation control algorithm proposed in this paper can effectively suppress the front wheel slip. Figure 6(e) shows the front axle torque distribution factors during the even distribution and dynamic distribution control, respectively. It can be seen in the figure that as the front axle load decreases, the target torque allocated to the front axle also decreases. Figure 6(f) shows that the test vehicle using the dynamic distribution torque control algorithm can smoothly climb a steep slope of $50 \%$, while the test vehicle that does not use the algorithm fails to climb.
4.2. Actual Vehicle Test. Based on the test platform of the four-wheel hub motor-driven off-road vehicle, the real-vehicle tests of mass and gradient estimation algorithms and front and rear axle dynamic compensation torque distribution control strategy are carried out.

The vehicle related parameters are shown in Table 1.

The parameters related to hub motor are shown in Table 2.

The physical photo of the in-wheel motor assembly system is shown in Figure 7.

In order to test the key state data of the vehicle, the sensor configuration of the vehicle is shown in Figure 8.

A $50 \%$ of the gradient climbing test is performed in the standard test site, and the standard test ramp is shown in Figure 9.

A longitudinal accelerometer is installed at the center of vehicle mass to instantly measure the vehicle longitudinal acceleration. Meanwhile, the parameter estimation algorithm is loaded into the vehicle controller for real-time operation, which is taken as an input parameter for parameter estimation and torque dynamic distribution control [20]. The test results are shown in Figures 10-12.

Results of $50 \%$ of the gradient climbing test are as follows. According to the test results, when the designed progressive RLS estimation algorithm operates on a real vehicle, it can accurately estimate the vehicle mass and road gradient in a real-time. As can be seen from real-time torque curve of each wheel of the front and rear axles, at $2.3 \mathrm{~s}$, the estimation algorithm accurately estimates that the road gradient is greater than $16^{\circ}$, and the front and rear axle driving force distribution coefficient is switched to $40: 60$ 

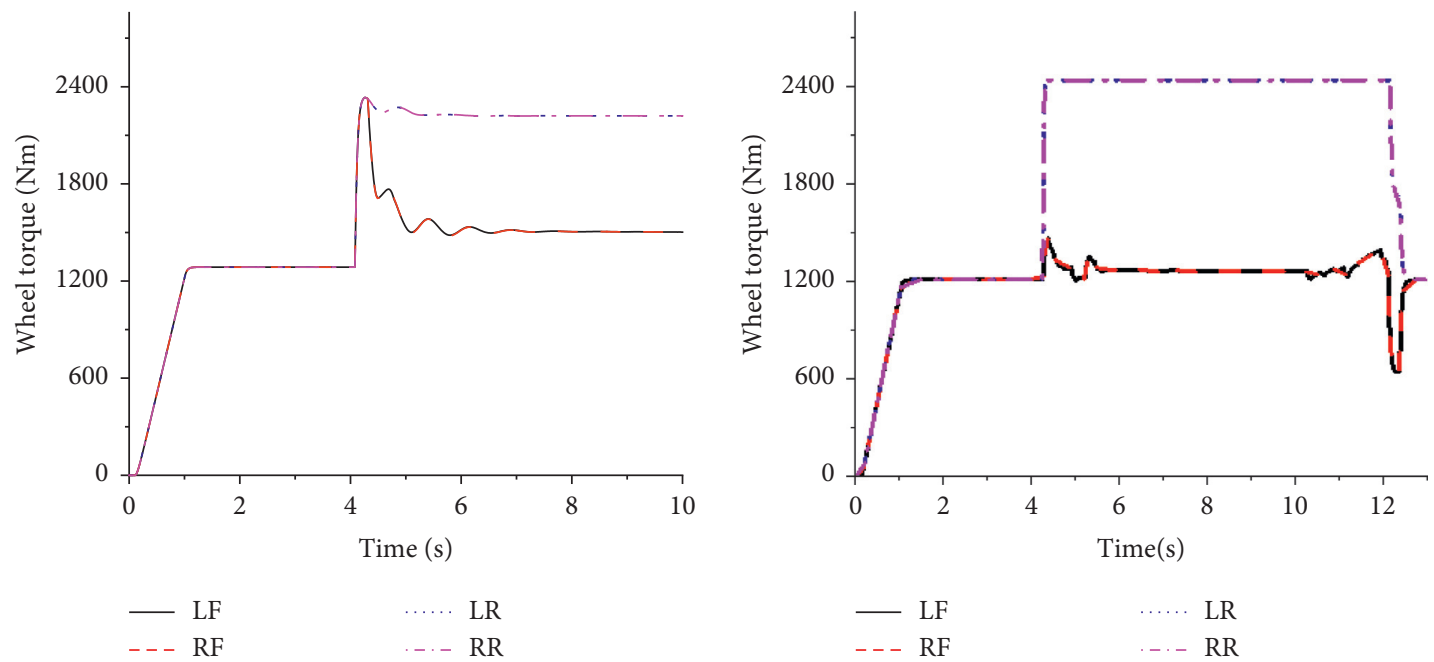

(a)

(b)
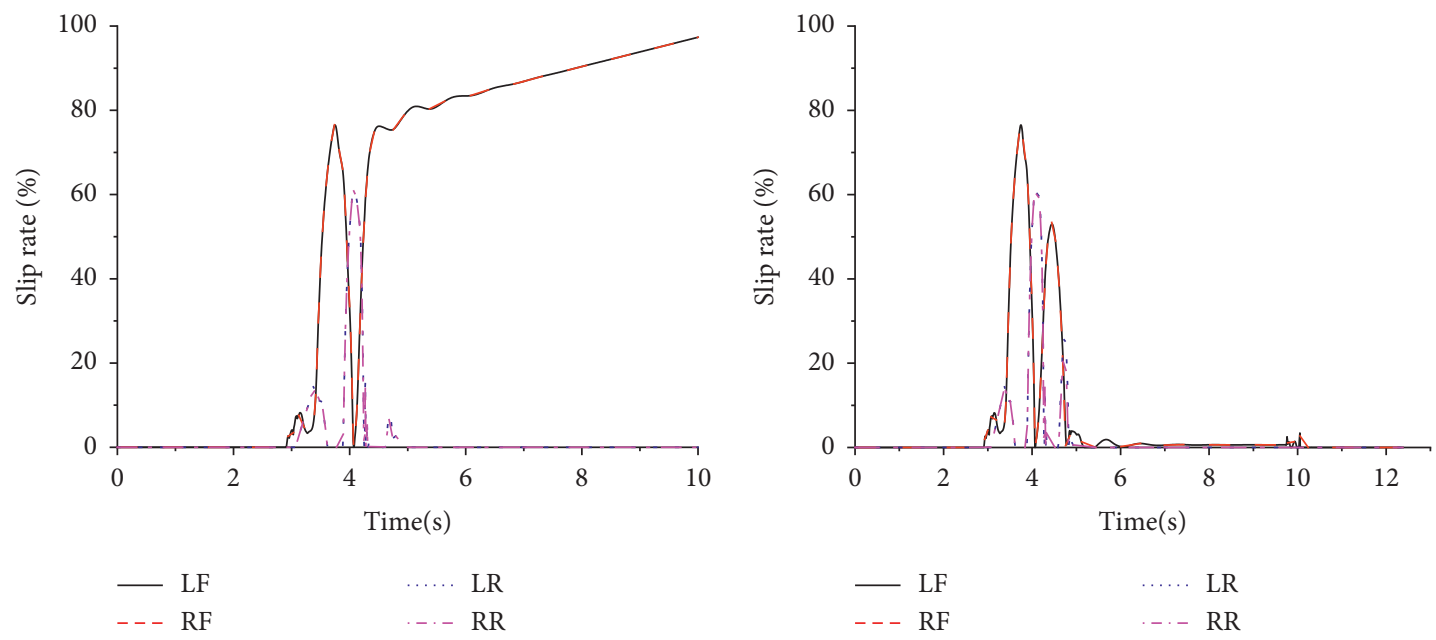

(c)

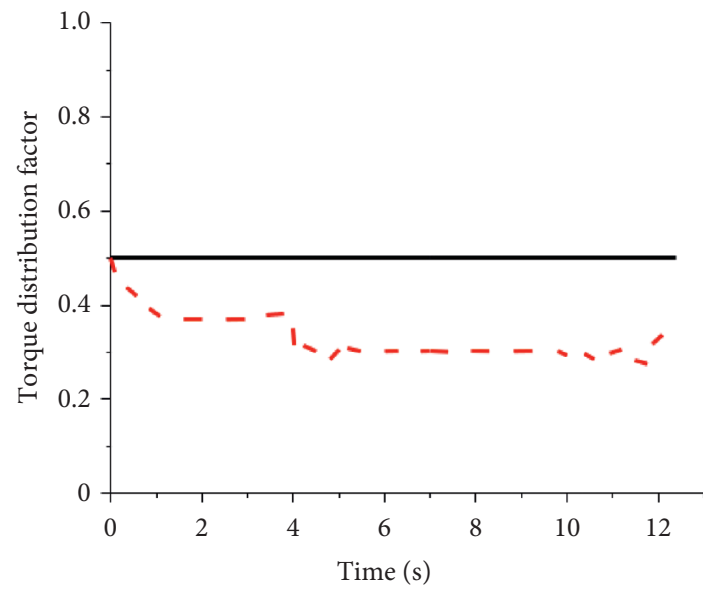

(d)

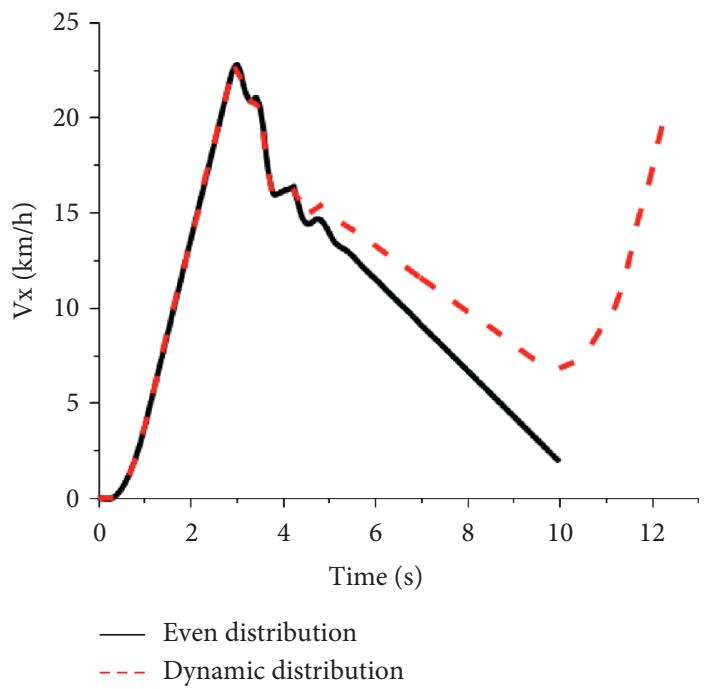

(f)

FiguRE 6: 50\% slope driving simulation. (a) Even distribution-wheel torque. (b) Dynamic distribution-wheel torque. (c) Even distributionslip rate. (d) Dynamic distribution-slip rate. (e) Front axle torque distribution factor. (f) Longitudinal speed comparison. 
TABLE 1: Vehicle parameters.

\begin{tabular}{lc}
\hline Vehicle parameters & Value \\
\hline Curb weight $(\mathrm{kg})$ & 2500 \\
Gross vehicle weight $(\mathrm{kg})$ & 3500 \\
Wheelbase $(\mathrm{mm})$ & 2946 \\
Center of mass height $(\mathrm{mm})$ & 781 \\
Distance from center of mass to front axle $(\mathrm{m})$ & 1.33 \\
Distance from center of mass to rear axle $(\mathrm{m})$ & 1.616 \\
Frontal area $\left(\mathrm{m}^{2}\right)$ & 2.4 \\
Reduction ratio & 5 \\
\hline
\end{tabular}

TABLE 2: Motor parameters.

\begin{tabular}{lc}
\hline Motor parameters & Value \\
\hline Rated/peak power $(\mathrm{kW})$ & $35 / 70$ \\
Rated/peak speed (RPM) & $1670 / 3500$ \\
Rated/peak torque (N.m) & $200 / 500$ \\
\hline
\end{tabular}

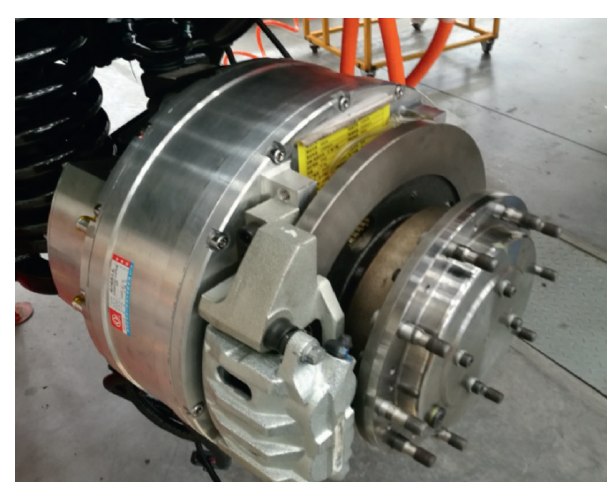

FIgURE 7: In-wheel motor assembly system.

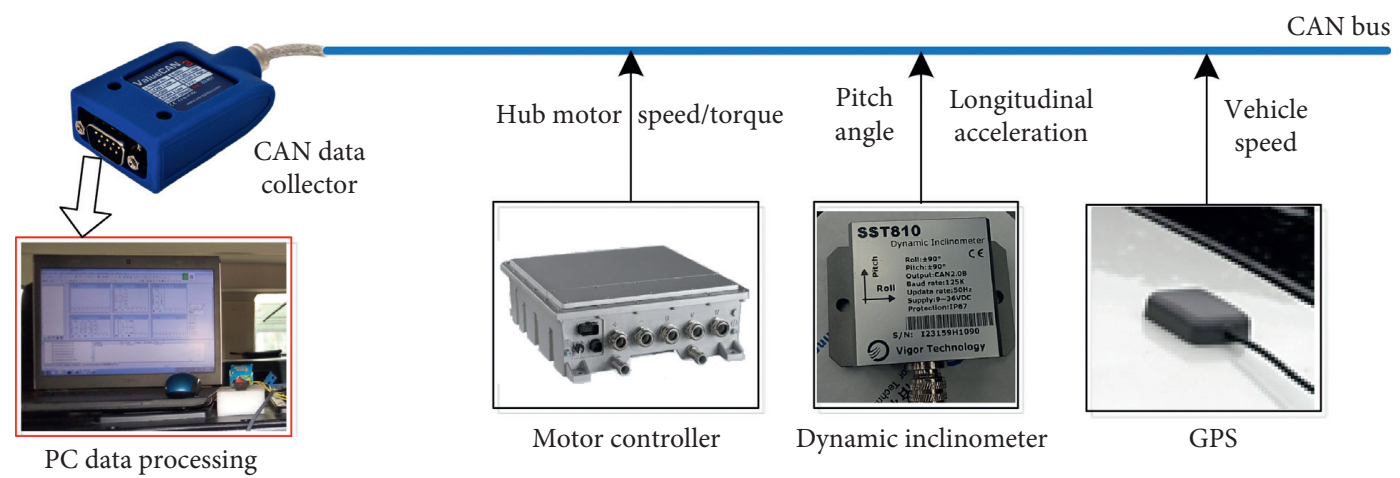

FIgURE 8: The sensor configuration.

accordingly; at $4.6 \mathrm{~s}$, the road gradient is estimated greater than $26^{\circ}$ so that the front and rear axle driving torque distribution coefficient is switched to $30: 70$. At the same time, the vehicle driving torque distribution coefficient takes into account the changes in vehicle longitudinal acceleration to timely compensate the torque distribution coefficients of the front and rear axles. Therefore, the dynamic self-compensation torque distribution strategy of the front and rear axles not only realizes the real-time control of the front and rear axle torque but also greatly enhances the power performance and trafficability of the whole vehicle. 


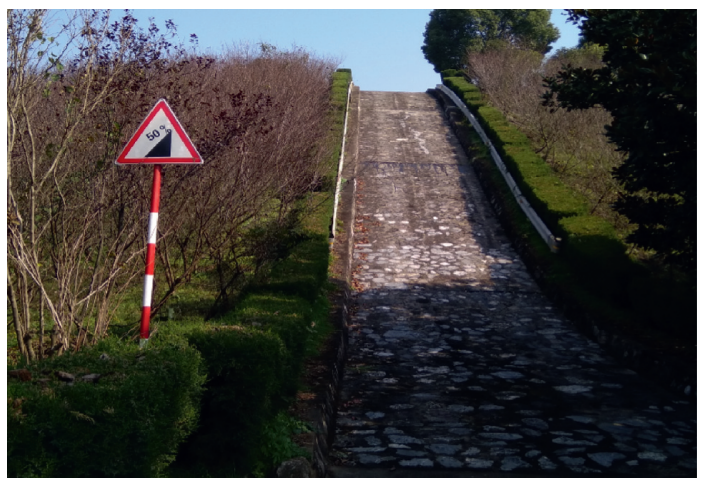

FIgURE 9: Standard test ramp.

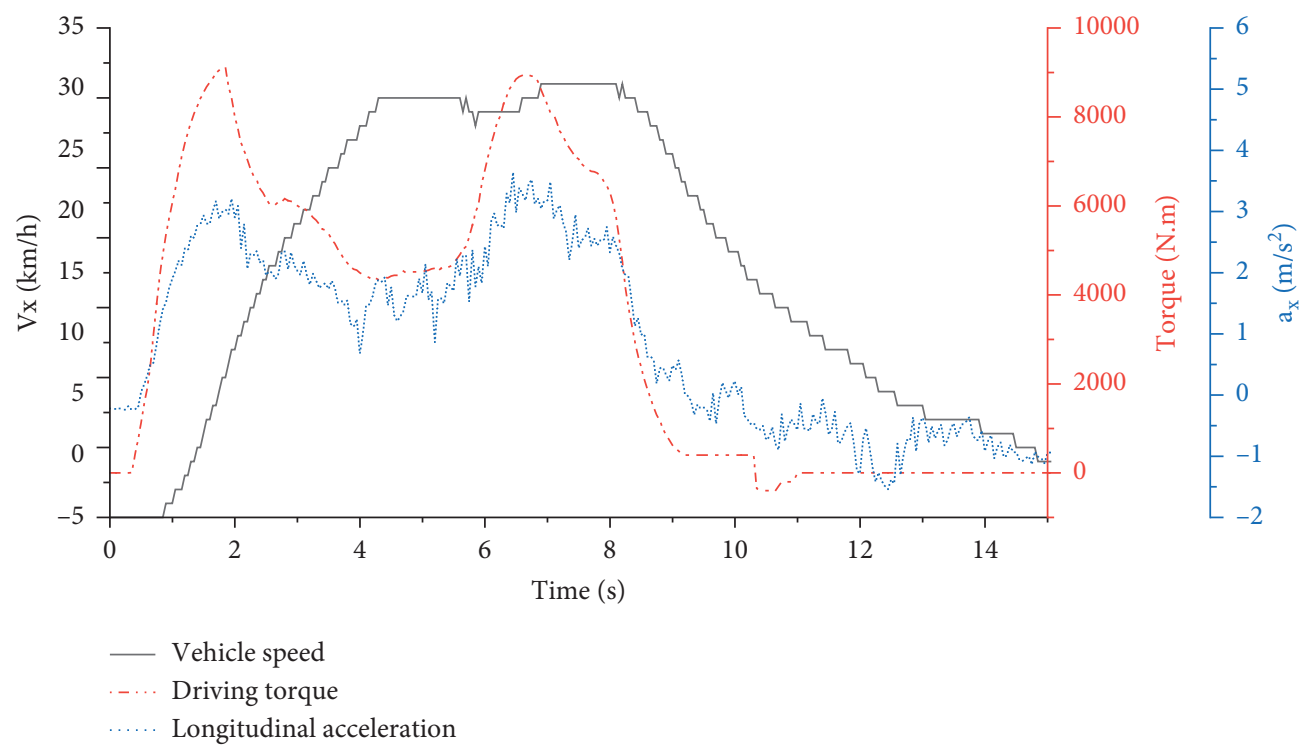

Figure 10: Vehicle speed, longitudinal acceleration, and driving torque.

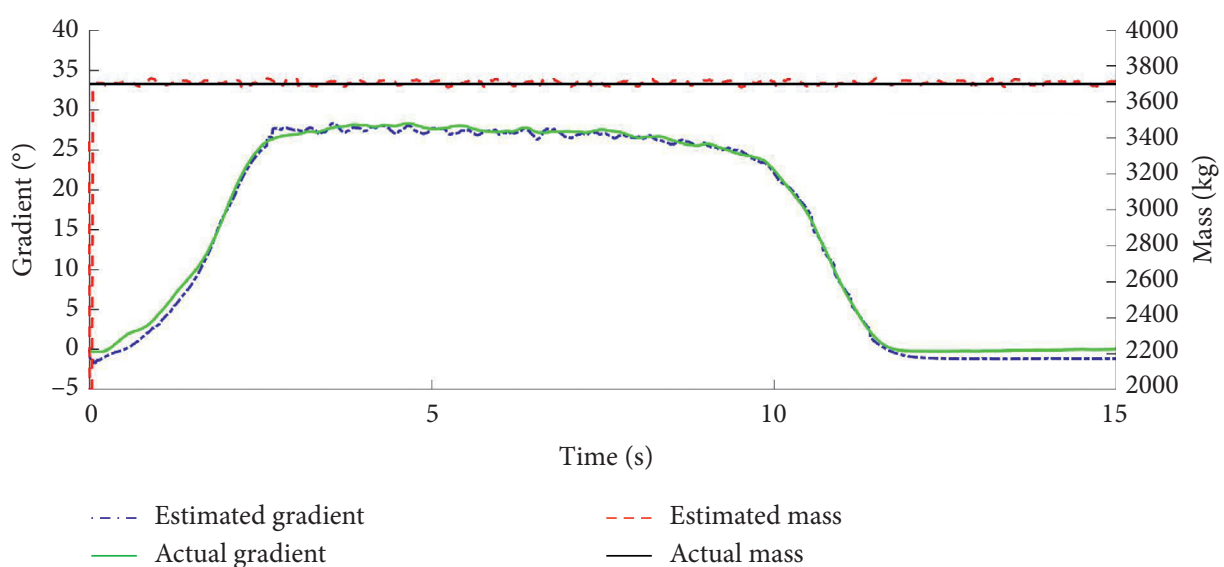

Figure 11: Estimation results of vehicle quality and road slope. 


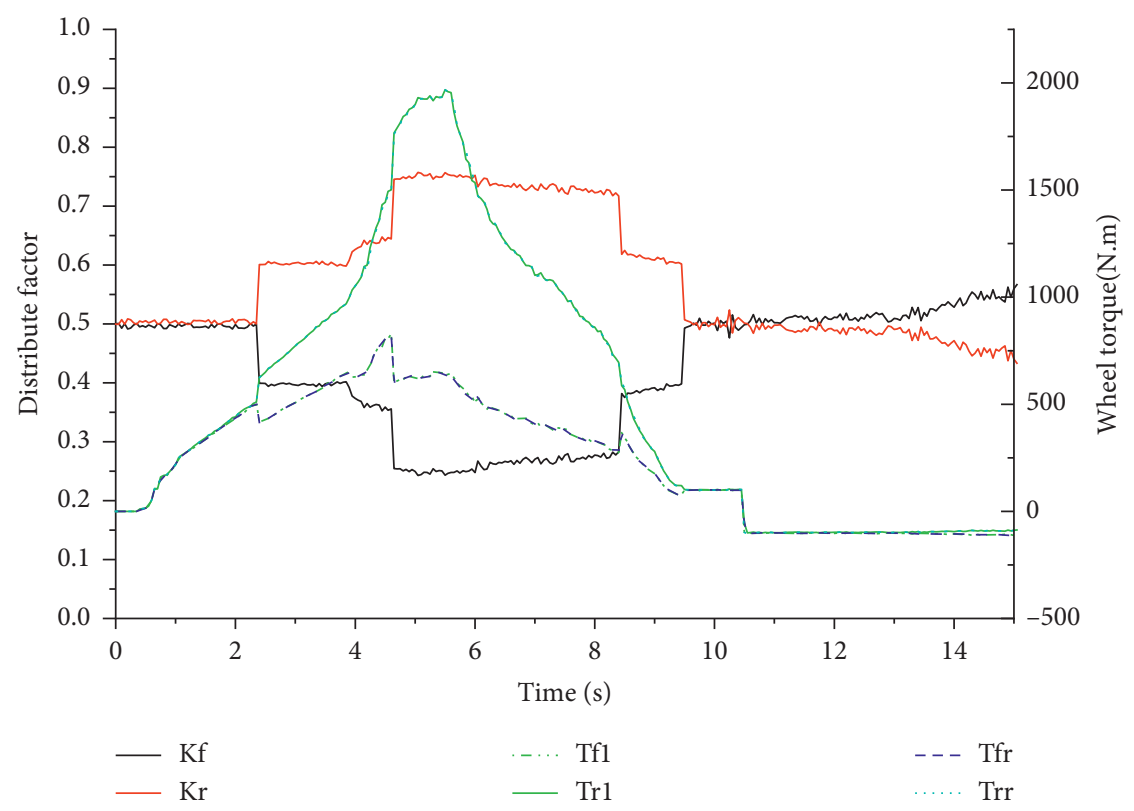

FIGURE 12: Torque distribution factor of front and rear axles and torque of each wheel.

\section{Conclusions}

In this paper, by means of focusing on a certain type of the four-wheel hub motor-driven off-road vehicle and making full use of the hub motor vehicle, a progressive RLS estimation algorithm is designed based on the vehicle longitudinal dynamics model, which can accurately estimate the vehicle mass as well as road gradient. Also, a dynamic selfcompensation torque distribution control strategy is proposed, which, through using estimated parameters and the vehicle longitudinal acceleration, realizes the dynamic distribution of the driving torque of vehicle front and rear axles and improves the vehicle trafficability and power performance. Besides, the CarSim and Matlab/Simulink joint simulation shows that the proposed progressive RLS estimation is more accurate in vehicle and road gradient estimation, and the estimated value can be taken as a reference for vehicle torque dynamic distribution. In the real-vehicle test, the results are consistent with the simulation, verifying the effectiveness of the proposed estimation algorithm and control strategy.

However, there is still some room for optimizing the designed control strategy. Specifically, in terms of driving torque distribution, vehicle longitudinal acceleration, road gradient, real-time vehicle dynamic parameters including yaw rate and roll angle, and road conditions such as road adhesion coefficient are all the parameters that need to be considered in the real-time control of the whole vehicle. In view of this, the future learning and research look into the changing law of each dynamic parameter and its application in the control of the whole vehicle.

\section{Data Availability}

The data used to support the findings of this study are available from the corresponding author upon request.

\section{Conflicts of Interest}

The authors declare that there are no conflicts of interest.

\section{Acknowledgments}

This study was funded by the Innovative Research Team Development Program of Ministry of Education of China (IRT_17R83) and the 111 Project (B17034) of China.

\section{References}

[1] G. Li and C. Zong, "Review on electric vehicle with four-wheel independent drive in-wheel motors," Journal of Liaoning University of Technology (Natural Science Edition), vol. 35, no. 1, pp. 47-52, 2014.

[2] M. Izhar Ishak, H. Ogino, and Y. Yamamoto, "Numerical simulation analysis of an oversteer in-wheel small electric vehicle integrated with four-wheel drive and independent steering," International Journal of Vehicular Technology, vol. 2016, Article ID 7235471, 12 pages, 2016.

[3] X. Jin, G. Yin, X. Zeng, and J. Chen, "Robust gain-scheduled output feedback yaw stability control for in-wheel-motordriven electric vehicles with external yaw-moment," Journal of the Franklin Institute, vol. 355, no. 18, pp. 9271-9297, 2018.

[4] Y.-H. Liu, T. Li, Y.-Y. Yang, X.-W. Ji, and J. Wu, "Estimation of tire-road friction coefficient based on combined APF-IEKF and iteration algorithm," Mechanical Systems and Signal Processing, vol. 88, pp. 25-35, 2017.

[5] R. Wang, H. Jing, C. Hu, F. Yan, and N. Chen, "Robust $H \infty$ path following control for autonomous ground vehicles with delay and data dropout," IEEE Transactions on Intelligent Transportation Systems, vol. 17, no. 7, pp. 2042-2050, 2016.

[6] D. Savitski, D. Schleinin, V. Ivanov et al., "Improvement of traction performance and off-road mobility for a vehicle with four individual electric motors: driving over icy road," Journal of Terramechanics, vol. 69, pp. 33-43, 2017. 
[7] B. Li, A. Goodarzi, A. Khajepour, S. K. Chen, and B. Litkouhi, "An optimal torque distribution control strategy for fourindependent wheel drive electric vehicles," Vehicle System Dynamics, vol. 53, no. 8, pp. 1172-1189, 2017.

[8] D. Tan, Q. Wang, and Y. Wu, "Modal analysis of in-wheel motor-driven electric vehicle based on bond graph theory," Shock and Vibration, vol. 2017, Article ID 6459154, 9 pages, 2017.

[9] Z. Shuai, H. Zhang, J. Wang, J. Li, and M. Ouyang, "Lateral motion control for four-wheel-independent-drive electric vehicles using optimal torque allocation and dynamic message priority scheduling," Control Engineering Practice, vol. 24, no. 1, pp. 55-66, 2014.

[10] X. Jin, J. Yang, Y. Li, B. Zhu, J. Wang, and G. Yin, “Online estimation of inertial parameter for lightweight electric vehicle using dual unscented Kalman filter approach," IET Intelligent Transport Systems, vol. 14, no. 5, pp. 412-422, 2020.

[11] B. Huang, S. Wu, S. Huang, and X. Fu, "Lateral stability control of four-wheel independent drive electric vehicles based on model predictive control," Mathematical Problems in Engineering, vol. 2018, Article ID 6080763, 15 pages, 2018.

[12] L. Chen, T. Chen, X. Xu, Y. Cai, H. Jiang, and X. Sun, "Multiobjective coordination control strategy of distributed drive electric vehicle by orientated tire force distribution method," IEEE Access, vol. 6, pp. 69559-69574, 2018.

[13] X. Jin, Z. Yan, G. Yin, S. Li, and C. Wei, "An adaptive motion planning technique for on-road autonomous driving," IEEE Access, vol. 9, pp. 2655-2664, 2021.

[14] X. Xia, L. Xiong, and Y.-Y. Hou, "Vehicle stability control based on driver's emergency alignment intention recognition," International Journal of Automotive Technology, vol. 18, no. 6, pp. 993-1006, 2017.

[15] P. Hang, X. Xia, and X. Chen, "Handling stability advancement with $4 \mathrm{WS}$ and DYC coordinated control: a gainscheduled robust control approach," IEEE Transactions on Vehicular Technology, vol. 70, no. 4, pp. 3164-3174, 2021.

[16] Z. Li, L. Zheng, W. Gao, and Z. Zhan, "Electromechanical coupling mechanism and control strategy for in-wheel-motor-driven electric vehicles," IEEE Transactions on Industrial Electronics, vol. 66, no. 6, pp. 4524-4533, 2019.

[17] Z. Yu, Y. Feng, and L. Xiong, "Review on vehicle dynamics control of distributed drive electric vehicle," Journal of Mechanical Engineering, vol. 49, no. 8, pp. 105-114, 2013.

[18] B. Ren, H. Chen, H. Zhao, and L. Yuan, "MPC-based yaw stability control in in-wheel-motored EV via active front steering and motor torque distribution," Mechatronics, vol. 38, pp. 103-114, 2016.

[19] Z. Wang, Y. Wang, L. Zhang, and M. Liu, "Vehicle stability enhancement through hierarchical control for a four-wheelindependently-actuated electric vehicle," Energies, vol. 10, no. 7, p. $947,2017$.

[20] M.-D. Gong, H.-H. Wang, and X. Wang, "Active suspension control based on estimated road class for off-road vehicle," Mathematical Problems in Engineering, vol. 2019, Article ID 3483710, 17 pages, 2019. 